Original Article

\title{
Neutrophil Elastase Inhibitor Sivelestat Attenuates Myocardial Injury after Cardioplegic Arrest in Rat Hearts
}

\author{
Masahiro Fujii, MD, PhD and Ryuzo Bessho, MD, PhD
}

\begin{abstract}
Purpose: Sivelestat, a neutrophil elastase inhibitor, attenuates global ischemia-induced myocardial damage and coronary endothelial dysfunction. Here, we investigated whether sivelestat exerts the cardioprotective effects against cardioplegic arrest in rat hearts. Methods: Isolated Langendorff-perfused rat hearts were randomly allocated to three groups and subjected to 2-min infusions with St. Thomas' Hospital cardioplegic solution No. 2 (STH2) and 30-min global ischemia followed by 60 -min reperfusion as follows: (i) control (STH2 treatment only), (ii) sivelestat $(19 \mu \mathrm{mol} / \mathrm{L})$ infusion for the first $10 \mathrm{~min}$ of reperfusion, and (iii) sivelestat $(19 \mu \mathrm{mol} / \mathrm{L})$ infusion for $10 \mathrm{~min}$ before ischemia and for the first $10 \mathrm{~min}$ of reperfusion. Left ventricular developed pressure (LVDP) recovery and troponin $\mathrm{T}$ leakage were measured at the end of reperfusion. Coronary flow response to acetylcholine (ACh) was assessed.

Results: Single and multiple doses of sivelestat significantly improved LVDP recovery $(69 \pm 15$ and $69 \pm 14$ vs $48 \pm 15$ [control]; $p<0.05)$ and decreased troponin $T$ leakage $(0.4 \pm 0.3$ and $0.7 \pm 0.5$ vs $1.7 \pm 0.6$ [control]; $p<0.05)$. Multiple doses of sivelestat significantly improved coronary flow response to ACh $(121 \pm 9$ vs $105 \pm 4 ; p<0.05)$.

Conclusions: Addition of sivelestat to $\mathrm{STH} 2$ attenuates myocardial injury after cardioplegic arrest in rat hearts. This cardioprotective effect was achieved even when sivelestat was administered during early reperfusion.
\end{abstract}

Keywords: sivelestat, cardioplegia, ischemia-reperfusion, myocardial protection, rat

\section{Introduction}

Recent advances in intraoperative myocardial cardioplegic protection have improved the post-ischemic hemodynamic function, attenuated the incidence of perioperative

Cardiovascular Surgery, Nippon Medical School Chiba Hokusoh Hospital, Inzai, Chiba, Japan

Received: September 1, 2019; Accepted: November 12, 2019 Corresponding author: Masahiro Fujii, MD, PhD. Cardiovascular Surgery, Nippon Medical School Chiba Hokusoh Hospital, 1715 Kamagari, Inzai, Chiba 270-1694, Japan

Email: m-fujii@nms.ac.jp

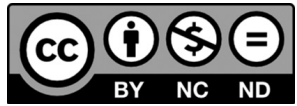

This work is licensed under a Creative Commons Attribution-NonCommercialNoDerivatives International License.

(C)2020 The Editorial Committee of Annals of Thoracic and Cardiovascular Surgery infarction, and decreased mortality. Nevertheless, although the use of conventional hyperkalemic cardioplegia is predominant, potassium cardioplegia has been reported to cause cytotoxicity and abnormalities in myocardial metabolism after cardioplegic arrest. ${ }^{1)}$ This has necessitated the use of protective additives in cardioplegic solutions and development of new intraoperative myocardial protection additives or alternatives to hyperkalemic solutions. ${ }^{2)}$

Sivelestat sodium hydrate is a specific neutrophil elastase inhibitor currently used for the treatment of acute lung injury (ALI) or acute respiratory distress syndrome (ARDS). ${ }^{3)}$ It is known to simultaneously attenuate ischemia and reperfusion injury in the lung. ${ }^{4)}$ Sivelestat has also been reported to attenuate neutrophil elastase or proinflammatory cytokines production and improve pulmonary dysfunction in patients undergoing extracorporeal circulation. ${ }^{5-7)}$ 
We previously showed that improved myocardial protection in response to simple global ischemia achieved via sivelestat administration during the early reperfusion period was similar to that achieved via its pre-ischemic administration. ${ }^{8)}$ We also suggested that one of mechanisms underlying the protective effect of sivelestat was the attenuation of coronary endothelial ischemia-reperfusion injury. ${ }^{8)}$ In this study, we investigated whether multiple doses of sivelestat could attenuate ischemia and/or reperfusion injury after cardioplegic arrest more effectively in the heart and enhance the myocardial protective effect of conventional hyperkalemic cardioplegia. Consequently, based on this, it might be possible to obtain a clinically applicable means to improve myocardial protection by cardioplegic arrest during cardiac surgery.

\section{Materials and methods}

\section{Animals}

Adult male Wistar rats (240-300 g body weight) were used (Saitama experiment animals, Saitama, Japan). All animals received humane care in compliance with the "Principles of Laboratory Animal Care" formulated by the National Society for Medical Research and the "Guide for the Care and Use of Laboratory Animals" prepared by the Institute of Laboratory Animal Research, published by the National Institute of Health (NIH Publication No. 85-23, revised 1996). Additionally, this study was approved by the Animal Ethics Committee of Nippon Medical School. The rats were anesthetized using pentobarbital $(50 \mathrm{mg} / \mathrm{kg}$, intraperitoneal) and anticoagulation was performed using heparin (1000 IU/kg, intravenous).

\section{Heart isolation and perfusion}

The hearts were quickly excised and immersed in cold $\left(4^{\circ} \mathrm{C}\right) \mathrm{Krebs}-\mathrm{Henseleit} \mathrm{bicarbonate} \mathrm{buffer}(\mathrm{KHB})$. The aorta was then cannulated, and the heart was perfused at $37^{\circ} \mathrm{C}$ in the Langendorff mode with KHB at a constant pressure $(75 \mathrm{mmHg})$, as previously described. ${ }^{8)}$ The heart was prepared by inserting a saline-filled vinyl balloon into the left ventricle through the left atrium, and the balloon was connected to a pressure transducer to measure the left ventricular pressure. The balloon volume was then adjusted to obtain a left ventricular end-diastolic pressure (LVEDP) of 3-8 $\mathrm{mmHg}$. All hearts were equilibrated via a 20 -min aerobic perfusion, and the following baseline readings were recorded: left ventricular systolic pressure (LVSP, mmHg), LVEDP ( $\mathrm{mmHg}$ ), heart rate

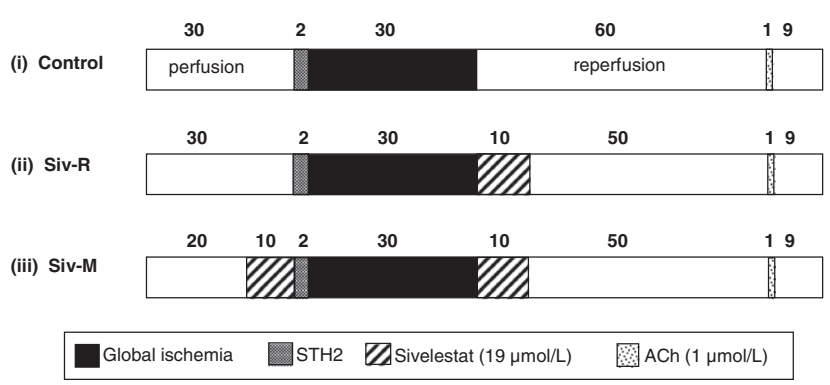

Fig. 1 Experimental perfusion protocol. The hearts were aerobically Langendorff-perfused with Krebs-Henseleit bicarbonate buffer at a constant pressure equivalent to $75 \mathrm{mmHg}$ before and after ischemia. (i) Control: 60-min reperfusion with KHB (only STH2 treatment) (ii) Siv-R: infusion with $19 \mu \mathrm{mol} / \mathrm{L}$ sivelestat-dissolved KHB for the first $10 \mathrm{~min}$ of reperfusion; (iii) Siv-M: infusion with $19 \mu \mathrm{mol} / \mathrm{L}$ sivelestatdissolved KHB for 10 min before ischemia and for the first $10 \mathrm{~min}$ of reperfusion. ACh: acetylcholine; STH2: St. Thomas' Hospital cardioplegic solution No. 2

(beats/min), and coronary flow ( $\mathrm{mL} / \mathrm{min})$. Left ventricular developed pressure (LVDP) was calculated by subtracting the LVEDP from the LVSP. The hearts with baseline values beyond the acceptable ranges of LVDP (>80 $\mathrm{mmHg}$ ), heart rate ( $>220$ beats/min), and coronary flow $(8-16 \mathrm{~mL} / \mathrm{min})$ were excluded.

\section{Perfusion medium}

KHB composition was as follows: $118.5 \mathrm{mmol} / \mathrm{L} \mathrm{NaCl}$; $25.0 \mathrm{mmol} / \mathrm{L} \mathrm{NaHCO}_{3} ; 4.8 \mathrm{mmol} / \mathrm{L} \mathrm{KCl} ; 1.2 \mathrm{mmol} / \mathrm{L}$ $\mathrm{MgSO}_{4} ; 1.18 \mathrm{mmol} / \mathrm{L} \mathrm{KH}_{2} \mathrm{PO}_{4} ; 1.4 \mathrm{mmol} / \mathrm{L} \mathrm{CaCl}_{2}$; and $11.0 \mathrm{mmol} / \mathrm{L}$ glucose. KHB was prepared daily and filtered through a $5-\mu \mathrm{m}$ cellulose nitrate filter. St. Thomas' Hospital cardioplegic solution No. 2 (STH2; Miotector®; Mochida Pharmaceutical Co., Tokyo, Japan), a conventional hyperkalemic cardioplegic solution, was prepared daily with the following composition: $110.0 \mathrm{mmol} / \mathrm{L} \mathrm{NaCl}$, $110.0 ; 16.0 \mathrm{mmol} / \mathrm{L} \mathrm{MgCl}_{2} \cdot 2 \mathrm{H}_{2} \mathrm{O} ; 16.0 \mathrm{mmol} / \mathrm{L} \mathrm{KCl}$; $1.2 \mathrm{mmol} / \mathrm{L} \mathrm{CaCl} 2 \cdot 2 \mathrm{H}_{2} \mathrm{O}$; and $10.0 \mathrm{mmol} / \mathrm{L} \mathrm{NaHCO}$. The $\mathrm{pH}$ was adjusted to 7.8 at $37^{\circ} \mathrm{C}$, and the solution was filtered through a $5-\mu \mathrm{m}$ cellulose nitrate filter before use. Sivelestat was dissolved in KHB $(10 \mu \mathrm{g} / \mathrm{mL})$ to obtain a final concentration of $19 \mu \mathrm{mol} / \mathrm{L}$; this concentration was reported to be effective for cardioprotection. ${ }^{8)}$ Acetylcholine $(\mathrm{ACh})$ chloride was dissolved in distilled water and diluted using oxygenated KHB to obtain $1 \mu \mathrm{mol} / \mathrm{L}$ ACh-dissolved KHB, as previously described.7)

\section{Perfusion protocol}

The perfusion protocol is shown in Fig 1. For all perfusion protocols, each heart was equilibrated via a 
Table 1 Baseline value and final percentage of recovery of heart rate, LVDP, and coronary flow and the final LVEDP values for Langendorff-perfused rat hearts

\begin{tabular}{lrrrr}
\hline & \multicolumn{1}{c}{ Control } & Siv-R & Siv-M & p value \\
\hline Heart rate & & & & \\
Baseline (beats/min) & $283.5 \pm 24.9$ & $297.2 \pm 28.9$ & $262.8 \pm 30.4$ & 0.138 \\
$\quad$ Recovery (\%) & $72.6 \pm 19.5$ & $93.2 \pm 4.8^{*}$ & $98.4 \pm 11.6^{* *}$ & 0.011 \\
LVDP & & & & \\
Baseline (mmHg) & $112.547 \pm 24.1$ & $109.8 \pm 16.8$ & $111.6 \pm 13.8$ & 0.966 \\
$\quad$ Recovery (\%) & $47.5 \pm 14.6$ & $69.4 \pm 15.4^{\#}$ & $68.9 \pm 14.4^{\# \#}$ & 0.034 \\
Coronary flow & & & & \\
Baseline (mL/min) & $15.3 \pm 3.9$ & $15.1 \pm 4.7$ & $15.3 \pm 0.9$ & 0.991 \\
$\quad$ Recovery (\%) & $59.4 \pm 9.7$ & $86.0 \pm 22.0^{\text {b }}$ & $69.3 \pm 16.7$ & 0.046 \\
LVEDP & & & & \\
Baseline (mmHg) & $5.4 \pm 1.9$ & $5.6 \pm 2.3$ & $6.2 \pm 1.2$ & 0.787 \\
$\quad$ Recovery (mmHg) & $34.9 \pm 14.9$ & $27.6 \pm 14.5$ & $34.2 \pm 15.9$ & 0.664 \\
\hline
\end{tabular}

Control, additional 10-min perfusion with KHB (treatment only with St. Thomas' Hospital cardioplegic solution No. 2); Siv-R, infusion with $19 \mu \mathrm{mol} / \mathrm{L}$ sivelestat-dissolved KHB for the first $10 \mathrm{~min}$ of reperfusion; Siv-M, infusion with $19 \mu \mathrm{mol} / \mathrm{L}$ sivelestat-dissolved KHB for $10 \mathrm{~min}$ before ischemia and for the first 10 min of reperfusion. KHB: Krebs-Henseleit bicarbonate buffer; LVDP: left ventricular developed pressure; LVEDP: left ventricular end diastolic pressure $* \mathrm{p}=0.032$ vs. control, ${ }^{*} * \mathrm{p}=0.009$ vs. control, ${ }^{\#} \mathrm{p}=0.040$ vs. control, ${ }^{\# \#} \mathrm{p}=0.044$ vs. control, ${ }^{b} \mathrm{p}=0.029$ vs. control.

20-min aerobic $\mathrm{KHB}$ perfusion at $37^{\circ} \mathrm{C}$. After equilibration, the hearts were randomly divided into the following three groups ( $\mathrm{n}=6$ per group) and then subjected to a 2-min STH2 infusion and 30 -min global $\left(37^{\circ} \mathrm{C}\right)$ ischemia, followed by a 60-min reperfusion: (i) control group, 60-min reperfusion with KHB (only STH2 treatment) (ii) reperfusion-sivelestat group (Siv-R), infusion with $19 \mu \mathrm{mol} / \mathrm{L}$ sivelestat-dissolved KHB for the first $10 \mathrm{~min}$ of reperfusion; (iii) multiple-sivelestat group (Siv-M): infusion with $19 \mu \mathrm{mol} / \mathrm{L}$ sivelestat-dissolved KHB for $10 \mathrm{~min}$ before ischemia and for the first $10 \mathrm{~min}$ of reperfusion.

\section{Myocardial function}

Reperfusion was performed for $60 \mathrm{~min}$, as described. The final recovery of myocardial function was measured during and after reperfusion.

\section{Myocardial injury}

To evaluate myocardial damage, the coronary effluents were collected during reperfusion and total troponin $\mathrm{T}$ leakage was measured via an electrochemiluminescence immunoassay. ${ }^{9)}$

\section{Endothelial function}

The coronary flow in all hearts was measured, $1 \mu \mathrm{mol} / \mathrm{L}$ ACh was infused for $1 \mathrm{~min}$, and coronary flow changes were recorded for the subsequent $10 \mathrm{~min}$. As a constant perfusion pressure was maintained, the increase and decrease in coronary flow were considered to reflect relaxation and constriction of the coronary vasculature, respectively.

\section{Statistical analysis}

Post-ischemic recovery of LVDP, heart rate, and coronary flow were expressed as a percentage of baseline values; LVEDP and troponin $\mathrm{T}$ were expressed as an absolute value (LVEDP: $\mathrm{mmHg}$; troponin $\mathrm{T}: \mu \mathrm{g} / \mathrm{g}$ wet weight). Coronary flow changes after ACh infusion were expressed as a percentage of the baseline values. All data are expressed as mean \pm standard deviation (SD). Continuous variables among three groups were compared for significance using the one-way analysis of variance (ANOVA) or two-way repeated-measures ANOVA with correction by linear regression, as appropriate. If significance was established, post-hoc analysis was performed using the Dunnett's test, which allowed for multiple comparisons. All statistical tests were two-tailed; $\mathrm{p}<0.05$ was considered to indicate statistical significance. All statistical analyses were performed using JMP version 10.0 (SAS Inc., Cary, NC, USA).

\section{Results}

\section{Recovery of myocardial function}

Baseline and final recovery measurements for all parameters are shown in Table 1. The control hearts showed an 
a

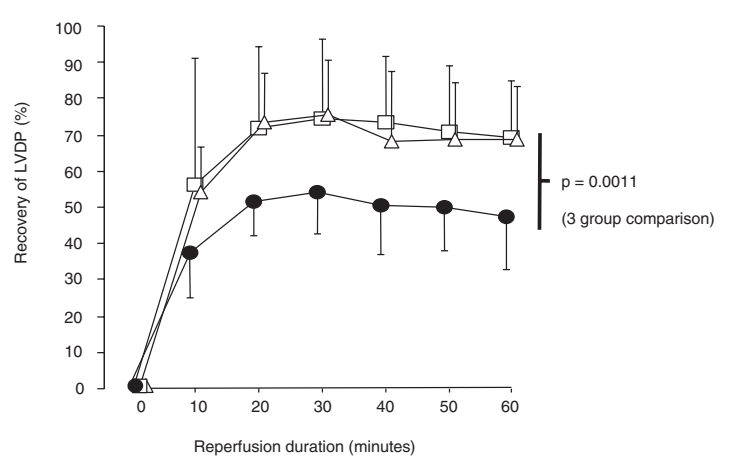

b

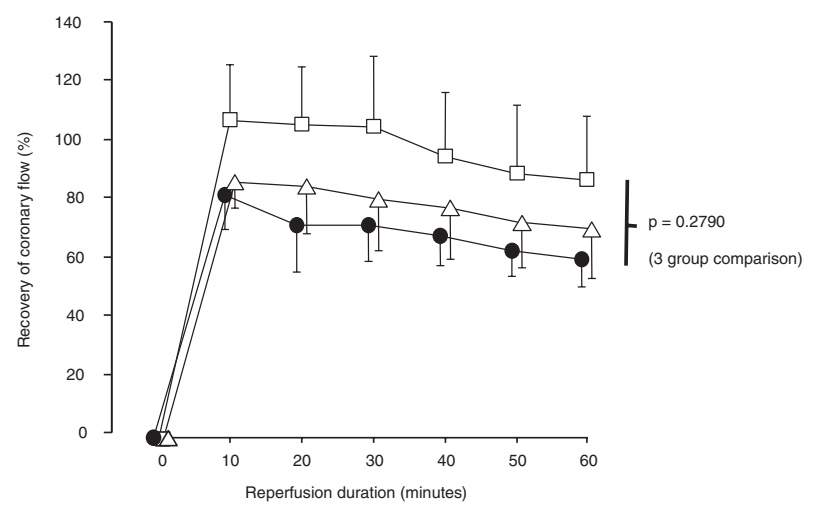

Fig. 2 Changes in recovery of (a) LVDP (expressed as a percentage of the baseline value) and (b) coronary flow (expressed as a percentage of the baseline value) during 60 -min of reperfusion. Values represent mean \pm SD ( $n=6$ hearts/group). Filled circle: Control, 60-min reperfusion with KHB (STH2 treatment only); open square: Siv-R, infusion with $19 \mu \mathrm{mol} / \mathrm{L}$ sivelestat-dissolved KHB for the first $10 \mathrm{~min}$ of reperfusion; open triangle: Siv-M, infusion with $19 \mu \mathrm{mol} / \mathrm{L}$ sivelestat-dissolved KHB for $10 \mathrm{~min}$ before ischemia and for the first $10 \mathrm{~min}$ of reperfusion. KHB: Krebs-Henseleit bicarbonate buffer; LVDP: left ventricular developed pressure; STH2: St. Thomas' Hospital cardioplegic solution No. 2

LVDP recovery of approximately $50 \%$ of that in the pre-ischemic condition. The hearts in the other two groups recovered rapidly to values higher than those in the control group, and the values reached a plateau within $20 \mathrm{~min}$; however, there were no significant differences between the two groups at different time points (Fig. 2a). The final recovery of LVDP in the Siv-M and Siv-R groups was significantly higher than that in the control group (Table 1). At the end of ischemia, LVEDP values were elevated to $50-60 \mathrm{mmHg}$ compared to baseline levels, but the differences in LVEDP values among the groups were not statistically significant. These values gradually decreased during reperfusion and reached $30-40 \mathrm{mmHg}$ at the end of reperfusion in all groups.

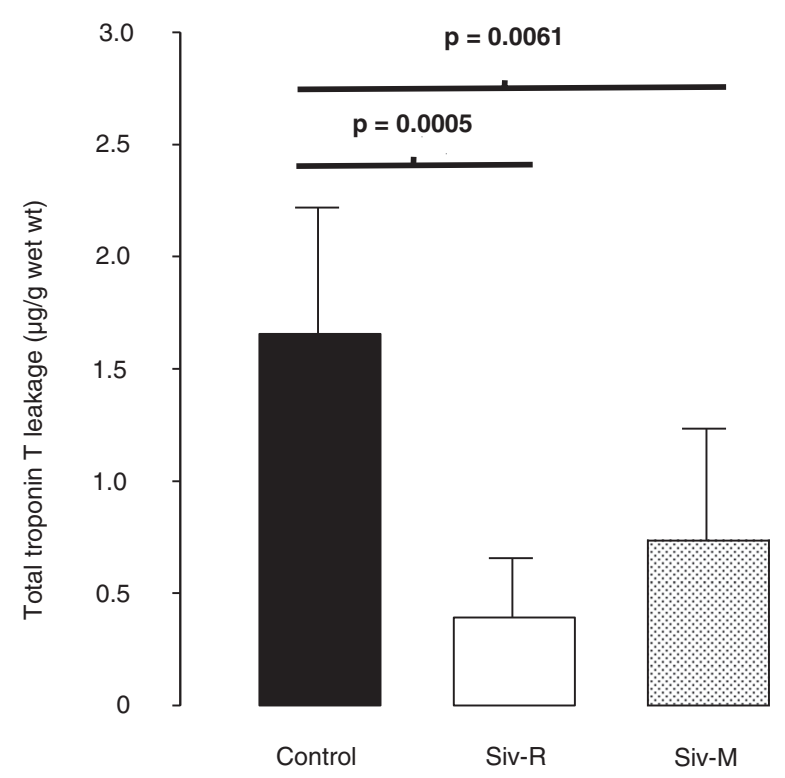

Fig. 3 Total troponin $\mathrm{T}$ leakage (expressed as $\mu \mathrm{g} / \mathrm{g}$ wet weight) during $60 \mathrm{~min}$ of reperfusion. Values represent mean $\pm \mathrm{SD}$ ( $\mathrm{n}=6$ hearts/group). (i) Control: 60-min reperfusion with KHB (STH2 treatment only) (ii) Siv-R: infusion with $19 \mu \mathrm{mol} / \mathrm{L}$ sivelestatdissolved KHB for the first $10 \mathrm{~min}$ of reperfusion; (iii) Siv-M: infusion with $19 \mu \mathrm{mol} / \mathrm{L}$ sivelestatdissolved $\mathrm{KHB}$ for $10 \mathrm{~min}$ before ischemia and for the first 10 minutes of reperfusion. KHB: KrebsHenseleit bicarbonate buffer; STH2: St. Thomas' Hospital cardioplegic solution No. 2

The coronary flow in the Siv-R group remained at a higher level than that in the control and Siv-M groups during reperfusion, but this did not reach significance (Fig. 2b). The final recovery of coronary flow in the Siv-R group was significantly higher than that in the control group, but not that in the Siv-M group.

\section{Myocardial injury}

A significant reduction in total troponin $\mathrm{T}$ leakage ( $\mu \mathrm{g} / \mathrm{g}$ wet weight) was observed in the Siv-R and Siv-M groups, compared to that in the control group (Fig. 3). The addition of sivelestat during the pre-ischemic period did not enhance the cardioprotective effect observed after sivelestat administration in the early reperfusion period.

\section{Endothelial function}

The increase in coronary flow after ACh infusion in the Siv-M group was significantly higher than that in the control group (Fig. 4). Similarly, compared to that in the control group, the Siv-R group also showed an improvement in coronary flow after ACh infusion; however, the difference was not statistically significant (Fig. 4). 


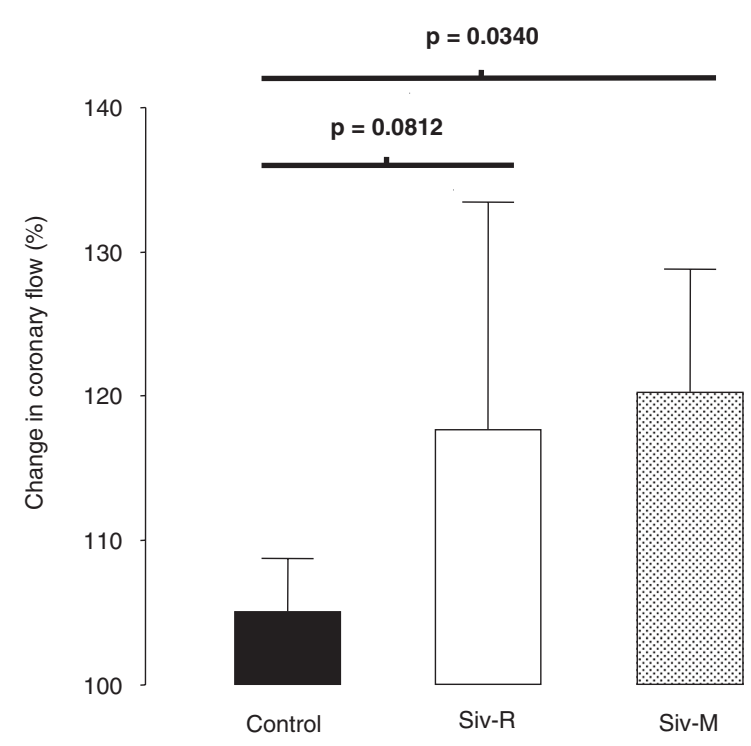

Fig. 4 The response of coronary flow to ACh. Height of the bar indicates the increase in coronary flow expressed as a percentage of the baseline values (measured just before ACh administration). Values represent mean $\pm \mathrm{SD}$ ( $\mathrm{n}=6$ hearts/group). (i) Control group: 60-min reperfusion with KHB (STH2 treatment only) (ii) Siv-R: infusion with $19 \mu \mathrm{mol} / \mathrm{L}$ sivelestatdissolved KHB for the first $10 \mathrm{~min}$ of reperfusion; (iii) Siv-M: infusion with $19 \mu \mathrm{mol} / \mathrm{L}$ sivelestatdissolved KHB for 10 min before ischemia and for the first $10 \mathrm{~min}$ of reperfusion. ACh: acetylcholine; KHB: Krebs-Henseleit bicarbonate buffer; STH2: St. Thomas' Hospital cardioplegic solution No. 2

\section{Discussion}

Neutrophil infiltration and the release of neutrophil elastase play a central role in ALI/ARDS and cause increased vascular permeability, which leads to alveolar injury and interstitial edema. ${ }^{10)}$ Sivelestat inhibits neutrophil elastase, preserves neutrophil deformability, and improves pulmonary microcirculation in humans and animals. ${ }^{11,12)}$ The Guidelines for Treatment of ALI/ ARDS by the Japanese Respiratory Society recommend sivelestat for ALI treatment. ${ }^{13)}$ Interestingly, excess surgical stress leads to high perioperative mortality, which is mainly caused by systemic inflammatory response syndrome (SIRS). The lung is the primary target organ of cytokine hyperproduction during SIRS. Intravenous sivelestat administration attenuates the perioperative inflammatory response and improves postoperative pulmonary function in patients undergoing esophagectomy, ${ }^{14)}$ liver resection, ${ }^{15)}$ and aortic surgery ${ }^{16)}$ and in neonatal, infant, and pediatric patients undergoing cardiac surgery. ${ }^{17)}$
Recently, sivelestat was also reported to exert protective effects against re-expansion pulmonary edema, which is one of the crucial complications that occurs after minimal invasive cardiac surgery with minithoracotomy. ${ }^{18)}$ Most clinical studies have demonstrated that sivelestat attenuates lung injury or improves pulmonary dysfunction; however, Toyama et al. showed that it improves not only the respiratory index but also changes in the fractional area of the left ventricle during pediatric cardiovascular surgery with cardiopulmonary bypass. ${ }^{17)}$ Consistently, the present study demonstrated an improvement in both LV function and endothelial function after cardioplegic arrest when sivelestat was administered during the first $10 \mathrm{~min}$ of reperfusion in isolated Langendorff-perfused rat hearts.

Myocardial protection during cardiac surgery has improved due to the development of hyperkalemic cardioplegia, which induces a depolarized heart arrest. Despite this clinical success, the development of additives for intraoperative myocardial protection or alternatives to hyperkalemic solution has been proposed. The coronary endothelium has been identified as an important organ that locally regulates coronary perfusion by producing vasoactive substances. ${ }^{19)}$ Not only ischemiareperfusion injury but also exposure of the coronary endothelium to hyperkalemic cardioplegia has harmful effects on coronary endothelial function. ${ }^{20-22)}$ Gohra et al. demonstrated that nitric oxide (NO) release from the coronary vasculature is significantly decreased during cardiac arrest through the repetitive administration of hyperkalemic cardioplegia. ${ }^{23)}$ Furthermore, Métais et al. reported that NO production decreases due to the downregulation of endothelial NO synthase (eNOS) caused by cardioplegia-reperfusion. ${ }^{24)}$ Interestingly, sivelestat was suggested to prevent eNOS uncoupling, thereby reducing superoxide overproduction and preserving NO production. ${ }^{25)}$ Furthermore, NOS inhibition was found to diminish the functional cardioprotective effect of sivelestat, confirming that sivelestat reduces tissue superoxide generation. Consistently, we previously reported that sivelestat significantly preserves endothelium-dependent vasorelaxation after ischemia-reperfusion. ${ }^{8)}$ Here, we showed that it results in similar endothelial preservation when administered after crystalloid hyperkalemic cardioplegic arrest.

In the present study, we evaluated the cardioprotective efficacy of the neutrophil elastase inhibitor, sivelestat, after cardioplegic arrest in an isolated rat model. To the best of our knowledge, this is the first study to demonstrate an additive cardioprotective effect of neutrophil 
elastase inhibition with STH2. Our findings indicate that sivelestat enhances the protective effects of hyperkalemic cardioplegia during cardiac surgery. However, multiple doses of sivelestat did not further improve cardioprotection compared to that with a single dose of sivelestat during early reperfusion. In our previous study, ${ }^{8)}$ the recovery of LVDP did not differ significantly regardless of whether sivelestat was administered before or after global ischemia. Although we expected that multiple doses of sivelestat would provide more effective protection than administration in early reperfusion only, both groups showed similar results. Unlike that found in a previous study, pretreatment with sivelestat might be washed away by hyperkalemic cardioplegia before ischemia in this setting. Further research should be performed to address this.

Admittedly, it is true that there are a couple of limitations. Sivelestat is a specific neutrophil elastase inhibitor that attenuates ischemia-reperfusion injury by reducing neutrophil activity. However, in our study, the hearts were perfused with KHB instead of whole blood, and only a few neutrophils could have infiltrated the heart. Further studies using a Langendorff blood-perfused model are thus needed. Moreover, ischemic heart disease consists of a multifactorial process, and several types of myocardial damage influence the method of cardioprotection. Hearts used in the present study were obtained from normal rats, and the protective effect of sivelestat might differ in hearts suffering from ischemic injury or disease. Additionally, although any such hearts are likely to require prolonged cardiac arrest to repair the lesion, the duration of global ischemia used in our study was relatively short.

\section{Conclusions}

Addition of the neutrophil elastase inhibitor sivelestat enhances the myocardial protection provided by STH2 and attenuates ischemia-reperfusion injury-induced endothelial dysfunction in isolated rat hearts. The administration of sivelestat both before and after cardioplegic arrest did not further improve cardioprotection compared to that with a single dose of sivelestat during early reperfusion. Sivelestat administration during early reperfusion might thus attenuate myocardial injury and improve surgical outcomes in the clinical setting.

\section{Acknowledgments}

We wish to thank Dr. Kazutora Mizukami (PhD in Statistical Science, President of Medical Data Management,
Fukuoka, Japan) for statistical assistance. We are grateful to Dr. David J Chambers (Cardiac Surgical Research, The Rayne Institute, St Thomas' Hospital, London, UK) for academic advice. We would also like to thank Editage (www.editage.com) for English language editing.

\section{Disclosure Statement}

The authors have no conflicts of interest to disclose.

\section{References}

1) Sellke FW, Shafique T, Schoen FJ, et al. Impaired endothelium-dependent coronary microvascular relaxation after cold potassium cardioplegia and reperfusion. J Thorac Cardiovasc Surg 1993; 105: 52-8.

2) Chambers DJ, Hearse DJ. Developments in cardioprotection: "polarized" arrest as an alternative to "depolarized" arrest. Ann Thorac Surg 1999; 68: 1960-6.

3) Aikawa N, Ishizaka A, Hirasawa H, et al. Reevaluation of the efficacy and safety of the neutrophil elastase inhibitor, sivelestat, for the treatment of acute lung injury associated with systemic inflammatory response syndrome; a phase IV study. Pulm Pharmacol Ther 2011; 24: 549-54.

4) Ishikawa N, Oda M, Kawaguchi M, et al. The effects of a specific neutrophil elastase inhibitor (ONO-5046) in pulmonary ischemia-reperfusion injury. Transpl Int 2003; 16: 341-6.

5) Abe T, Usui A, Oshima $H$, et al. A pilot randomized study of the neutrophil elastase inhibitor, sivelestat, in patients undergoing cardiac surgery. Interact Cardiovasc Thorac Surg 2009; 9: 236-40.

6) Kohira S, Oka N, Inoue N, et al. Effect of the neutrophil elastase inhibitor sivelestat on perioperative inflammatory response after pediatric heart surgery with cardiopulmonary bypass: a prospective randomized study. Artif Organs 2013; 37: 1027-33.

7) Fujii M, Miyagi Y, Bessho R, et al. Effect of a neutrophil elastase inhibitor on acute lung injury after cardiopulmonary bypass. Interact Cardiovasc Thorac Surg 2010; 10: 859-62.

8) Kambe M, Bessho R, Fujii M, et al. Sivelestat reduces myocardial ischemia and reperfusion injury in rat hearts even when administered after onset of myocardial ischemia. Interact Cardiovasc Thorac Surg 2009; 8: 629-34.

9) Dutra RF, Mendes RK, Lins da Silva V, et al. Surface plasmon resonance immunosensor for human cardiac troponin $\mathrm{T}$ based on self-assembled monolayer. J Pharm Biomed Anal 2007; 43: 1744-50.

10) Carden D, Xiao F, Moak C, et al. Neutrophil elastase promotes lung microvascular injury and proteolysis of endothelial cadherins. Am J Physiol 1998; 275: H385-92. 
11) Iba T, Kidokoro A, Fukunaga M, et al. Pretreatment of sivelestat sodium hydrate improves the lung microcirculation and alveolar damage in lipopolysaccharide-induced acute lung inflammation in hamsters. Shock 2006; 26: 95-8.

12) Inoue $Y$, Tanaka $H$, Ogura $H$, et al. A neutrophil elastase inhibitor, sivelestat, improves leukocyte deformability in patients with acute lung injury. J Trauma 2006; 60: 936-43; discussion 943.

13) Committee for the preparation of 2016 academic joint ARDS clinical practice guidelines. Treatment. ARDS Clinical Practice Guidelines 2016. Tokyo: Sogoigakusha, 2016; pp 104-5. (in Japanese)

14) Wang ZQ, Chen LQ, Yuan Y, et al. Effects of neutrophil elastase inhibitor in patients undergoing esophagectomy: a systematic review and meta-analysis. World J Gastroenterol 2015; 21: 3720-30.

15) Yamazaki S, Takayama T, Moriguchi M, et al. Neutrophil elastase inhibitor following liver resection: a matched cohort study. Hepat Mon 2015; 15: e31235.

16) Morimoto N, Morimoto K, Morimoto $Y$, et al. Sivelestat attenuates postoperative pulmonary dysfunction after total arch replacement under deep hypothermia. Eur J Cardiothorac Surg 2008; 34: 798-804.

17) Toyama S, Hatori F, Shimizu A, et al. A neutrophil elastase inhibitor, sivelestat, improved respiratory and cardiac function in pediatric cardiovascular surgery with cardiopulmonary bypass. J Anesth 2008; 22: 341-6.

18) Yamashiro S, Arakaki R, Kise $Y$, et al. Prevention of pulmonary edema after minimally invasive cardiac surgery with mini-thoracotomy using neutrophil elastase inhibitor. Ann Thorac Cardiovasc Surg 2018; 24: 32-9.

19) Yang Q, Yu CM, He GW, et al. Protection of coronary endothelial function during cardiac surgery: potential of targeting endothelial ion channels in cardioprotection. Biomed Res Int 2014; 2014: 324364.

20) Saldanha C, Hearse DJ. Cardioplegia and vascular injury. Dissociation of the effects of ischemia from those of the cardioplegic solution. J Thorac Cardiovasc Surg 1994; 108: 279-90.

21) Keller MW, Geddes L, Spotnitz W, et al. Microcirculatory dysfunction following perfusion with hyperkalemic, hypothermic, cardioplegic solutions and blood reperfusion. Effects of adenosine. Circulation 1991; 84: $2485-94$.

22) Nilsson FN, Miller VM, Johnson CM, et al. Cardioplegia alters porcine coronary endothelial cell growth and responses to aggregating platelets. J Vasc Res 1993; 30: 43-52.

23) Gohra H, Fujimura Y, Hamano K, et al. Nitric oxide release from coronary vasculature before, during, and following cardioplegic arrest. World J Surg 1999; 23: 1249-53.

24) Métais C, Li J, Li J, et al. Serotonin-induced coronary contraction increases after blood cardioplegia-reperfusion: role of COX-2 expression. Circulation 1999; 100 (Suppl_2): II328-34.

25) Aune SE, Yeh ST, Kuppusamy P, et al. Sivelestat attenuates myocardial reperfusion injury during brief low flow postischemic infusion. Oxid Med Cell Longev 2013; 2013: 279847. 